\title{
Statistical Study of Variability in Rainfall and Analysis of Etreme Rainfall Events for Hill Stations of Uttarakhand
}

\author{
Bimal Pande, Sneh Joshi and Seema Pande* \\ Department of Physics (UGC-Centre of Advanced Study), Kumaun University, Nainital. \\ Email: pande.seema@yahoo.com
}

\begin{abstract}
Statistical analysis of rainfall pattern and its variability for 20 years (1990-2010) data is performed for two mountainous urban centres of Uttarakhand i.e. Almora $\left(29.6^{0} \mathrm{~N}, 79.67^{0} \mathrm{E}\right.$ and altitude $1,204 \mathrm{~m}$ asl $)$ and Nainital $\left(29.4^{\circ} \mathrm{N}, 79.47^{\circ} \mathrm{E}\right.$ and altitude 2,020m asl). Non Parametric method of Principal Component Analysis (PCA) gives the correlation between different extreme rainfall indices. It is concluded that PCA suggest $90 \%$ of the variance in composite matrix of extreme rainfall indices.
\end{abstract}

Keywords: Non-parametric methods, PCA, Rainfall patterns, Statistical analysis.

\section{Introduction}

The Himalayan region, being an orographically dominated region and in close proximity to northern hemispheric descending branch of the Hadley circulation, is characterized by its unique weather and climate. In particular, both orographically induced convective processes and large scale circulation are likely to play important roles in the Himalayan weather and climate. The convective forcing can be measured in terms of quantities like convective available potential energy (CAPE); the large scale circulation can be quantified in terms of convergence/divergence of horizontal wind. In addition, remote processes like sea surface temperature (SST) over the pacific (El Nino/ La Nina) may also affect the Himalayan weather through induced divergence.

In this paper we have made an attempt to analyze rainfall trend and correlation of different indices of extreme rainfall in Central Himalaya. An attempt is also made to look at local perception about rainfall trends and extreme events. It is expected that this study fill the knowledge gap in testing and establishing interrelation of the extreme rainfall indices in mountainous terrain and geologically fragile areas. The study is conducted in two important mountain urban centres, i.e., Almora $\left(29.60^{\circ} \mathrm{N}, 79.67^{\circ} \mathrm{E}\right.$ and altitude $1204 \mathrm{~m}$ asl) and Nainital $\left(29.40^{\circ} \mathrm{N}\right.$, $79.47^{\circ} \mathrm{E}$ and altitude $2020 \mathrm{~m}$ asl) located in geologically fragile region of Central Himalayas, India. The study area falls in the Lesser Himalayan domain and experience a temperate climate. Mean monthly temperature in summer varies from $19^{\circ} \mathrm{C}$ to $24^{\circ} \mathrm{C}$. The rainfall varies widely in this region like other mountainous area. Its quantity to a large extent depends on the location of the place to the windward or leeward side of the high ridges. The entire area is influenced by the southwest monsoon, which arrives in the later parts of the June and continues till the end of the September. 70 to $80 \%$ of the total annual rainfall occurs during this period.

\section{Method and Data used}

The annual rainfall data (1990-2010) of Almora and Nainital are collected from government observatory (Vivekanand Parvatiya Krishi Anusandhan Sansthan, Almora) and (Aryabhatta Research Institute of Observational Sciences, Nainital), 
respectively (Figure 1). Nonparametric methods, i.e., Sen's method and the MannKendall test are used for determination of trend as these methods are less influenced by the presence of outliers in the data. Details of these methods are given by Guhathakurta et al. (2006) and Kumar et al. (2008). Both stations have different climatic conditions and mean of annual rainfall is significantly different $\left(\mathrm{t}_{\mathrm{stat}}=14.82 ; \mathrm{df}=13\right)$.

Principal component analysis (PCA) is conducted using SYSTAT 9 (SPSS Inc.) on the matrix of time series data of 19 indices to determine underlying temporal variance structure and check the correlation through time for each station. PCA is variable reduction procedure. It is useful when we have large number of variables and there is some redundancy in those variables. Because of this redundancy, it is possible to reduce the observed variables into a smaller number of principal components (artificial variables) that will account for most of the variance in the observed variables. PCA attempts to explain the overall variance in a data set by isolating a number of components with respect to newly defined axes, each of which corresponds to a variable (Richman 1986; Preisendorfer 1988; Graham 1988). The number of components extracted using the correlation matrix in a PCA is equal to the number of observed variables being analysed. The first component extracted in a PCA, accounts for a maximal amount of total variance in the observed variables. The second component will be correlated with some of the observed variables and it will be uncorrelated with the first component. It means correlation between component-1 and component- 2 would be zero. The remaining components account for a maximal amount of variance in the observed variables that was not accounted by the preceding components and is uncorrelated with all the preceding components. Thus, resulting components will display varying degree of correlation with the observed variables and are completely uncorrelated with one another.

\section{Results and Discussion}

The PCA has been attempted on the $14 \times 19$ matrix to determine the temporal variance in the extreme rainfall indices for both stations. The rotated and unrotated solutions are similar and according to Kaiser's criterion the cut-off value for the eigen values is at the eigen value number 3 (Eigen value $\geq 1.00$ ) (Kaiser 1959). These first three components accounted for $85 \%$ of the total variance for Almora, whose first principal component $\left(\mathrm{PC}_{1}\right)$ explains about $48 \%$ of the variance with absolute highest loading (>0.90) on the intensity indices i.e., mean of four largest events, mean of events above $95^{\text {th }}$ percentile, total annual rainfall divided by rainy days, mean of largest 5\% and total annual rainfall. Other intensity indices are also significantly correlated as indicated by the loadings of $\mathrm{PC}_{1}$. The time series of $\mathrm{PC}_{1}$ scores, which shows temporal variance in the largest of the extreme events and component scores, show decreasing trend $(\mathrm{p}=0.16$; not significant at 95\% confidence level) over the period 1990 to 2010 in Almora and this decrease in $\mathrm{PC}_{1}$ is recorded after 1993 (Figure. 2(a)). The second principal component $\left(\mathrm{PC}_{2}\right)$ explains about $26 \%$ of the variance in the composite matrix and had high positive loading on the total rainfall of four largest events divided by total annual rainfall. Time series of $\mathrm{PC}_{2}$ scores, which appears to characterize variance in the extreme per cent indices shows increasing trend $(\mathrm{p}=0.58$; not significant at 95\% confidence level) in case of Almora with a jump in 1999 (Fig. 2(b)). The third principal 
component $\left(\mathrm{PC}_{3}\right)$ is explaining only $11 \%$ of the total variance with the highest loading on consecutive rainy days (Figure 2(c)). Time series of $\mathrm{PC}_{3}$ scores also shows increasing trend $(\mathrm{p}=0.62 ;$ not significant at $95 \%$ confidence level) with jump in 2002.The larger variance in frequency and intensity indicators are correlated as explained by $\mathrm{PC}_{1}$ but, extreme per cent indicators are independent. This indicates that the increase in rainfall intensity may accompany the increase in frequency of extreme events. The decreasing trend in time series of $\mathrm{PC}_{1}$ means decline in frequency $(>10 \mathrm{~mm},>25.4 \mathrm{~mm}$ and $>100 \mathrm{~mm} /$ day) and intensity (all classes) of the rainfall events. Increasing trend in $\mathrm{PC}_{2}$ indicates rise in extreme per cent (all classes) possibly due to decline in annual rainfall at Almora during the study period. The $\mathrm{PC}_{1}$ component loading for Nainital explains $51 \%$ of variance with absolute highest loading (> 0.90) on intensity indices i.e., largest one day, mean of four largest events, mean of largest $5 \%$, mean of events above $95^{\text {th }}$ percentile and mean of events above $99^{\text {th }}$ percentile. $\mathrm{PC}_{1}$ shows decreasing trend over the period 19902010 and indicate decline in intensity indices; $\mathrm{p}=0.54$ (not significant at $95 \%$ confidence level; Fig. 2(a)). On the other hand, $\mathrm{PC}_{2}$ explained about $25 \%$ of the variance in the composite matrix and had higher positive loading on frequency indices. Time series analysis shows an increasing trend $(\mathrm{p}=0.77$; not significant at $95 \%$ confidence level) in $\mathrm{PC}_{2}$ (Fig. 2(b)). This means increasing trend in rainy days in Nainital is mainly in the form of low to medium intensity rainfall. $\mathrm{PC}_{3}$ explains only $12 \%$ of the total variance with highest loading on the frequency of daily events $>99^{\text {th }}$ percentile. Time series analysis shows an increasing trend in $\mathrm{PC}_{3}(\mathrm{p}=0.40$; not significant at $95 \%$ confidence level) (Fig 2(c)). In case of Nainital, intensity indicators and extreme per cent indicators are correlated, but frequency indicator is independent to intensity except for frequency $>100 \mathrm{~mm}$. The intensity indices of rainfall of extreme events and extreme per cent are decreasing, whereas frequency of rainfall may be increasing.

4. Conclusion: Daily rainfall data are analyzed for two urban centers of central Himalayan Region showing significantly different rainfall pattern during 1990-2010. The detection of trends using nonparametric methods i.e. Sen's method and the MannKendall test, show a decline in annual and monsoon rainfall at both stations. The perception of local people also confirms change in rainfall pattern in form of reduction in annual and monsoon rainfall and variation of rainfall pattern. PCA suggest that 3 principal components in the data can explain about $85 \%$ to $88 \%$ of the variance in the composite matrix of extreme rainfall indices in such cases. The loadings further reveal that first principal component explains about half of the variance in both the stations, but the time series and pattern of component loadings are different for both stations. In case of Almora, the variations in frequency and intensity indicators are correlated and show a decreasing trend. In case of Nainital, intensity indicators and frequency indicators are independent except for frequency $>100 \mathrm{~mm}$. This shows that the intensity of rainfall of extreme events and extreme per cent are decreasing, whereas frequency of low to medium intensity events may be increasing in Nainital. The differences in variance of two stations prompt toward isolated analysis of extreme events in different locations of Himalaya and any regionalization of results in climatologically diverse region may not provide correct pattern of extreme rainfall events. The results of the study can be used to 
identify the relation between recorded natural hazards and extreme rainfall events at these urban centre's and designing the monitoring network for forecasting possible future hazards.

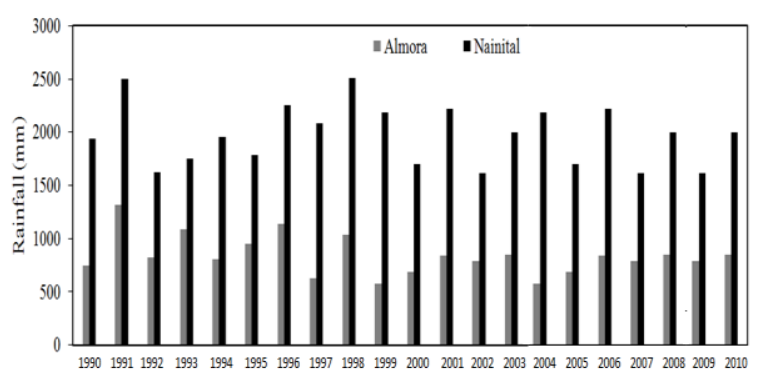

Fig 1: Annual Rainfall Data for Almora and Nainital

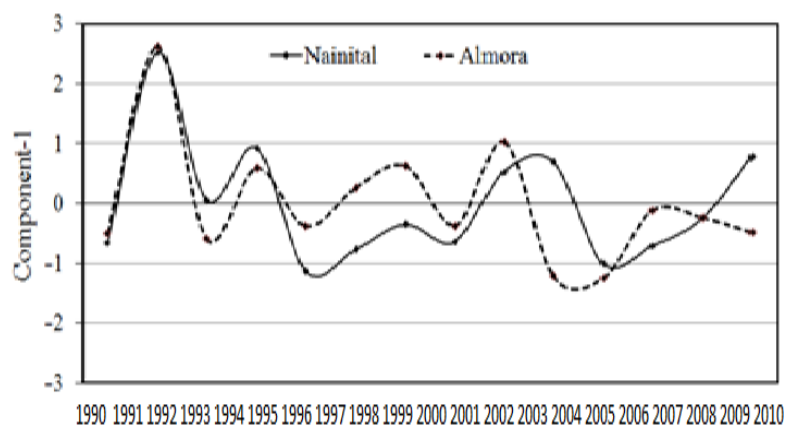

Fig 2 (a): PC1 for Almora and Nainital from 1990-2010

\section{References:}

Guhathakurta, P. and Rajeevan, M. (2006) Trends in the rainfall pattern over India. National Climate Centre Research Report No: 2/2006.

Kumar, K., Joshi, S. and Joshi, V. (2008) Climate Variability, Vulnerability and Coping Mechanism in Alaknanda Catchment, Central Himalaya. AMBIO 32(4): 286-291.

Richman, M.B. (1986), Journal of Climatology 6: 293-335.

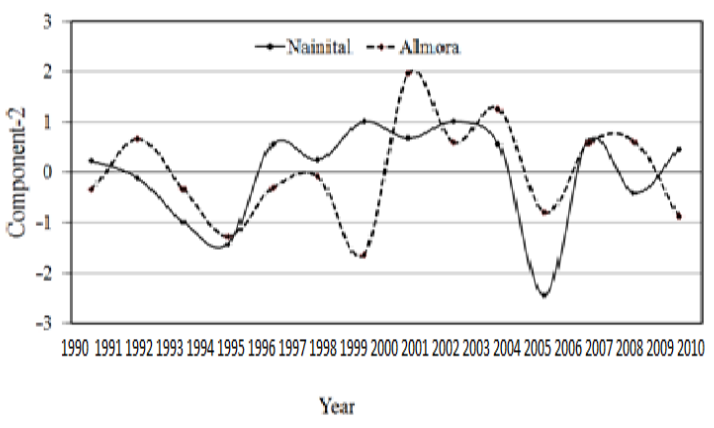

Fig 2 (b): : PC2 for Almora and Nainital from 1990-2010

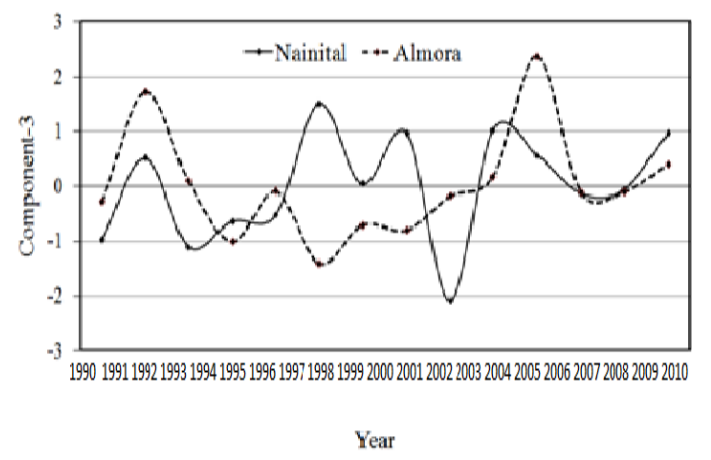

Fig 2(c) : PC3 for Almora and Nainital from 1990-2010.

Preisendorfer, R.W. (1988) Principal Component Analysis in Meteorology and Oceanography, Elsevier, New York, 425 pp.

Graham, S. (1988) Precipitation: Process and Analysis, Wiley, 455pp.

Kaiser, H.F. (1959) Computer program for varimax rotation in factor analysis. Psychological Measurement 19: 413-420. 